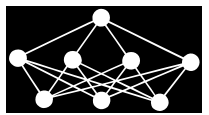

\title{
NOVEL STOCHASTIC STABILITY CONDITIONS OF FUZZY NEURAL NETWORKS WITH MARKOVIAN JUMPING PARAMETERS UNDER IMPULSIVE PERTURBATIONS
}

\author{
Y. Zhang, C.-D. Zheng
}

\begin{abstract}
This paper investigates the stochastic stability of fuzzy neural networks with Markovian jumping parameters and mixed delays under impulsive perturbations in mean square. The mixed delays consist of time-varying delay and continuously distributed delay. By employing a new Lyapunov-Krasovskii functional, linear convex combination technique, a novel reciprocal convex lemma and the free-weight matrix method, two novel sufficient conditions are derived to ensure the stochastic asymptotic stability of the equilibrium point of the considered networks in mean square. The proposed results, which are expressed in terms of linear matrix inequalities, can be easily checked via Matlab LMI Toolbox. Finally, two numerical examples are given to demonstrate the effectiveness and less conservativeness of our theoretical results over existing literature.
\end{abstract}

Key words: impulse, fuzzy neural networks, Markovian jumping, linear convex combination technique, reciprocal convex combination

Received: October 14, 2014

DOI: $10.14311 / \mathrm{NNW} .2016 .26 .001$

Revised and accepted: May 5, 2015

\section{Introduction}

In the last decades, cellular neural networks have been extensively studied and applied in many different fields such as associative memory, signal processing and some optimization problems. In such applications, it is of prime importance to ensure that the designed neural networks are stable. In practice, due to the finite speeds of the switching and transmission of signals, time delays do exist in a working network and thus should be incorporated into the model equation. In recent years, the dynamical behaviors of cellular neural networks with constant delays or timevarying delays or distributed delays have been studied by many researchers; see, for example, $[7,9,10,18,19,23,24,28,30]$ and the references therein.

Nevertheless, besides delay effects, impulsive effects likewise exist in a wide variety of evolutionary processes in which states are changed abruptly at certain

Yue Zhang, Cheng-De Zheng - Corresponding author, School of Science, Dalian Jiaotong University, Dalian, 116028, P. R. China, E-mail: 15566913851@163.com 
moments of time, involving such fields as medicine and biology, economics, mechanics, etc. There are many interesting results about impulsive neural networks up to date, e.g., Ref. $[3,21,26,31]$. Since impulsive perturbations can affect dynamical behaviors of the system just as time delays, it is meaningful to consider both time delays and impulsive effects of neural networks.

Fuzzy logic theory has been shown to be an appealing and efficient approach for dealing with the analysis and synthesis problems of complex nonlinear systems. In 1996, based on traditional cellular neural networks, Yang et al. introduced fuzzy cellular neural networks $[22,25,27]$, which combine fuzzy logic with the structure of traditional cellular neural networks and maintain local connectedness among cells. Unlike previous cellular neural network structures, fuzzy cellular neural networks have fuzzy logic between its template and input and/or output besides the sum of product operation, which allows us to combine the low of fuzzy systems. Fuzzy cellular neural network is a useful paradigm for image processing problems and Euclidean distance transformation. In addition, fuzzy cellular neural network has inherent connection to mathematical morphology, which is a cornerstone in image processing and pattern recognition. Recently, various interesting results on the stability and other behaviors of fuzzy cellular neural network have been reported $[6,11]$.

On the other hand, systems with Marvokian jumps have been attracting increasing research attention. This class of systems are the hybrid ones with two components in the state. The first one refers to the mode, which is described by a continuous-time finite-state Markovian process, and the second one refers to the state which is represented by a system of differential equations. The Markovian jump systems have the advantage of modeling the dynamic systems subject to abrupt variation in their structures, such as component failures or repairs, sudden environmental disturbance, changing subsystem interconnections, and operating in different points of a nonlinear plant. Recently, there has been a growing interest in the study of neural networks with Markovian jumping parameters [12, 20, 29, 35]. However, to the best of our knowledge, there are few results reported about the stochastic stability of fuzzy neural networks with Markovian jumping parameters and mixed delays under impulsive perturbations up to today.

Motivated by above discussion, this paper discusses the stochastic stability of fuzzy neural networks with Markovian jumping parameters and mixed delays under impulsive perturbations in mean square. The mixed delays consist of timevarying delay and continuously distributed delay. By employing a new LyapunovKrasovskii functional, linear convex combination technique, a novel reciprocal convex lemma and the free-weight matrix method, two novel sufficient conditions are derived to ensure the stochastic asymptotic stability of the equilibrium point of the considered networks in mean square. The proposed results, which are expressed in terms of linear matrix inequalities, can be easily checked via Matlab LMI Toolbox. Finally, two numerical examples shall be given to demonstrate the effectiveness and less conservativeness of our theoretical results over existing literature.

Notation: Throughout this paper, let $\mathbb{Z}_{+}$denote the set of positive integers, $W^{T}, W^{-1}$ denote the transpose and the inverse of a square matrix $W$, respectively. $W>0(<0)$ denotes a positive (negative) definite symmetric matrix, $I$ denotes the identity matrix with compatible dimension, $0_{m \times n}$ denotes the $m \times n$ zero matrix, 
the symbol "*" denotes a block that is readily inferred by symmetry. The shorthand $\operatorname{col}\left\{M_{1}, M_{2}, \ldots, M_{k}\right\}$ denotes a column matrix with the matrices $M_{1}, M_{2}, \ldots, M_{k}$. $\operatorname{diag}\{\cdot\}$ stands for a diagonal or block-diagonal matrix, $\mathbb{N}=\{1,2, \ldots, n\}$. For $\tau>0, \mathcal{C}\left([-\tau, 0] ; \mathbb{R}^{n}\right)$ denotes the family of continuous functions $\phi$ from $[-\tau, 0]$ to $\mathbb{R}^{n}$ with the norm $\|\phi\|=\sup _{-\tau \leq s \leq 0}|\phi(s)|$. Moreover, let $(\Omega, \mathbb{F}, \mathbb{P})$ be a complete probability space with a filtration $\left\{\mathbb{F}_{t}\right\}_{t \geq 0}$ satisfying the usual conditions and $\mathbb{E}\{\cdot\}$ representing the mathematical expectation. Denote by $\mathcal{C}_{\mathbb{F}_{0}}^{p}\left([-\tau, 0] ; \mathbb{R}^{n}\right)$ the family of all bounded, $\mathbb{F}_{0}$-measurable, $\mathcal{C}\left([-\tau, 0] ; \mathbb{R}^{n}\right)$-valued random variables $\xi=\{\xi(s):-\tau \leq s \leq 0\}$ such that $\sup _{-\tau \leq s \leq 0} \mathbb{E}|\xi(s)|^{p}<\infty$. $\|\cdot\|$ stands for the Euclidean norm; matrices, if not explicitly stated, are assumed to have compatible dimensions.

\section{Problem description and preliminaries}

Fuzzy Markovian jumping recurrent neural network model with mixed delays under impulsive perturbations can be described by the following model:

$$
\left\{\begin{aligned}
& \dot{x}_{i}(t)=- d_{i}(\eta(t)) x_{i}(t)+\sum_{j=1}^{n} a_{i j}(\eta(t)) f_{j}\left(x_{j}(t)\right)+\sum_{j=1}^{n} b_{i j}(\eta(t)) f_{j}\left(x_{j}(t-\tau(t, \eta(t)))\right) \\
&+\sum_{j=1}^{n} c_{i j} \varrho_{j}+\chi_{i}+\bigwedge_{j=1}^{n} \alpha_{i j} \int_{-\infty}^{t} k_{j}(t-s) f_{j}\left(x_{j}(s)\right) \mathrm{d} s \\
&+\bigvee_{j=1}^{n} \beta_{i j} \int_{-\infty}^{t} k_{j}(t-s) f_{j}\left(x_{j}(s)\right) \mathrm{d} s+\bigwedge_{j=1}^{n} \sigma_{i j} \varrho_{j}+\bigvee_{j=1}^{n} \delta_{i j} \varrho_{j}, \quad t \neq t_{k}, \\
& \Delta x_{i}\left(t_{k}\right)=x_{i}\left(t_{k}\right)-x_{i}\left(t_{k}^{-}\right)=J_{i k}\left(x_{i}\left(t_{k}^{-}\right)\right), \quad k \in \mathbb{Z}_{+}, \\
& x_{i}(s)=\varphi_{i}(s), \quad s \in(-\infty, 0], \quad i \in \mathbb{N},
\end{aligned}\right.
$$

where $\alpha_{i j}, \beta_{i j}, \sigma_{i j}$ and $\delta_{i j}$ are elements of fuzzy feedback MIN template, fuzzy feedback MAX template, fuzzy feed-forward MIN template and fuzzy feed-forward MAX template, respectively. $a_{i j}(\eta(t))$ and $b_{i j}(\eta(t))$ are elements of feedback template and $c_{i j}$ are elements of feed-forward template. $\Lambda$ and $\bigvee$ denote the fuzzy AND and fuzzy OR operations, respectively. $x_{i}(t), \varrho_{j}$ and $\chi_{i}$ denote state, input and bias of the $i$-th neurons respectively, $d_{i}(\eta(t)) \geq 0$ is a constant. $\{\eta(t), t \geq 0\}$ is a homogeneous, finite-state Markovian process with right continuous trajectories and taking values in finite set $\mathcal{N}=\{1,2, \ldots, N\}$ based on given probability space $(\Omega, \mathbb{F}, \mathbb{P})$ and the initial model $\eta_{0}$. Let $\Pi=\left[\pi_{i j}\right]_{N \times N}$ denote the transition rate matrix with transition probability:

$$
\mathbb{P}(\eta(t+\delta)=j \mid \eta(t)=i)=\left\{\begin{array}{cc}
\pi_{i j} \delta+o(\delta), & i \neq j \\
1+\pi_{i i} \delta+o(\delta), & i=j
\end{array}\right.
$$

where $\delta>0, \lim _{\delta \rightarrow 0^{+}} \frac{o(\delta)}{\delta}=0$ and $\pi_{i j}$ is the transition rate from mode $i$ to mode $j$ satisfying $\pi_{i j} \geq 0$ for $i \neq j$ with

$$
\pi_{i i}=-\sum_{j=1, j \neq i}^{N} \pi_{i j}, \quad i, j \in \mathcal{N} .
$$


$f_{i}(\cdot)$ is the activation function, $\tau(t, \eta(t))$ is the transmission delay. $k_{j}(s) \geq 0$ is the feedback kernel and satisfies

$$
\int_{0}^{\infty} k_{j}(s) \mathrm{d} s=1, \quad j \in \mathbb{N}
$$

Function $\varphi_{i}(s)(i \in \mathbb{N})$ is continuous on $(-\infty, 0]$, the norm is defined by

$$
\|\varphi\|_{\infty}=\max \left\{\sup _{-\infty \leq s \leq 0}\|\varphi(s)\|, \sup _{-\infty \leq s \leq 0}\|\dot{\varphi}(s)\|\right\} .
$$

In this paper, we make the following assumptions

(H1) The transmission delay $\tau(t, \eta(t))$ is time-varying and satisfies $0 \leq \tau(t, \eta(t)) \leq$ $\bar{\tau}(\eta(t)) \leq \bar{\tau}, \dot{\tau}(t, \eta(t)) \leq \mu(\eta(t))<1$, where $\bar{\tau}(\eta(t)), \bar{\tau}, \mu(\eta(t))$ are known constants.

(H2) The activation function $f(x(t))=\left(f_{1}\left(x_{1}(t)\right), f_{2}\left(x_{2}(t)\right), \ldots, f_{n}\left(x_{n}(t)\right)\right)^{T} \in \mathbb{R}^{n}$ is bounded and satisfies the following condition

$$
\lambda_{j}^{-} \leq \frac{f_{j}(\xi)-f_{j}(\zeta)}{\xi-\zeta} \leq \lambda_{j}^{+}, \quad \forall \xi, \zeta \in \mathbb{R}, \xi \neq \zeta
$$

where $\lambda_{j}^{-}, \lambda_{j}^{+}$are known real constants.

For simplicity, we denote $\Lambda_{1}=\operatorname{diag}\left\{\lambda_{1}^{-} \lambda_{1}^{+}, \lambda_{2}^{-} \lambda_{2}^{+}, \cdots, \lambda_{n}^{-} \lambda_{n}^{+}\right\}, \Lambda_{2}=\frac{1}{2} \operatorname{diag}\left\{\lambda_{1}^{-}\right.$ $\left.+\lambda_{1}^{+}, \lambda_{2}^{-}+\lambda_{2}^{+}, \cdots, \lambda_{n}^{-}+\lambda_{n}^{+}\right\}, \Lambda_{3}=\operatorname{diag}\left\{\lambda_{1}^{-}, \lambda_{2}^{-}, \cdots, \lambda_{n}^{-}\right\}, \Lambda_{4}=\operatorname{diag}\left\{\lambda_{1}, \lambda_{2}\right.$, $\left.\cdots, \lambda_{n}\right\}$, where $\lambda_{i}=\max \left\{\left|\lambda_{i}^{-}\right|,\left|\lambda_{i}^{+}\right|\right\}, \quad i=1, \ldots, n$.

(H3) Every function $J_{k}(x(t))=\left[J_{1 k}\left(x_{1}(t)\right), J_{2 k}\left(x_{2}(t)\right), \ldots, J_{n k}\left(x_{n}(t)\right)\right]^{T}: \mathbb{R}^{n} \rightarrow$ $\mathbb{R}^{n}$ is continuous for any $x(t)=\left(x_{1}(t), x_{2}(t), \ldots, x_{n}(t)\right)^{T} \in \mathbb{R}^{n}, k \in \mathbb{Z}_{+}$.

(H4) The impulsive time instants $t_{k}$ satisfy $0=t_{0}<t_{1}<\cdots<t_{k}<\cdots \rightarrow \infty$ and $\inf _{k \in \mathbb{Z}_{+}}\left\{t_{k}-t_{k-1}\right\}>0$.

For convenience, each possible value of $\eta(t)$ is denoted by $m(m \in \mathcal{N})$ in the sequel. Then we have

$$
\begin{gathered}
d_{i m}=d_{i}(\eta(t)), a_{i j m}=a_{i j}(\eta(t)), b_{i j m}=b_{i j}(\eta(t)), \tau_{m}(t)=\tau(t, \eta(t)), \\
\bar{\tau}_{m}=\bar{\tau}(\eta(t)), \mu_{m}=\mu(\eta(t)) .
\end{gathered}
$$

In addition, we use the following lemmas:

Lemma 1 (see [16]). Let $X, Y$ and $P$ be real matrices of appropriate dimensions with $P>0$. Then for any positive scalar $\varepsilon$ the following matrix inequality holds:

$$
X^{T} Y+Y^{T} X \leq \varepsilon^{-1} X^{T} P^{-1} X+\varepsilon Y^{T} P Y .
$$

Lemma 2 (see [22]). Let $x$ and $y$ be two states of system (1), then we have

$$
\begin{aligned}
& \left|\bigwedge_{j=1}^{n} \alpha_{i j} f_{j}\left(x_{j}\right)-\bigwedge_{j=1}^{n} \alpha_{i j} f_{j}\left(y_{j}\right)\right| \leq \sum_{j=1}^{n}\left|\alpha_{i j}\right|\left|f_{j}\left(x_{j}\right)-f_{j}\left(y_{j}\right)\right|, \\
& \left|\bigvee_{j=1}^{n} \beta_{i j} f_{j}\left(x_{j}\right)-\bigvee_{j=1}^{n} \beta_{i j} f_{j}\left(y_{j}\right)\right| \leq \sum_{j=1}^{n}\left|\beta_{i j}\right|\left|f_{j}\left(x_{j}\right)-f_{j}\left(y_{j}\right)\right| .
\end{aligned}
$$


Lemma 3 (see [11]). For any $x \in \mathbb{R}^{n}$, any constant matrix $A=\left[a_{i j}\right]_{n \times n}$ with $a_{i j} \geq 0$, the following matrix inequality holds:

$$
x^{T} A^{T} A x \leq n x^{T} A_{s}^{T} A_{s} x,
$$

where $A_{s}=\operatorname{diag}\left\{\sum_{i=1}^{n} a_{i 1}, \sum_{i=1}^{n} a_{i 2}, \ldots, \sum_{i=1}^{n} a_{i n}\right\}$.

Lemma 4 (see [8]). Let $z(t) \in \mathbb{R}^{n}$ have continuous derived function $\dot{z}(t)$ on interval $[a, a+\omega]$, then for any $n \times n-$ matrix $\Theta>0$, the following inequality holds:

$$
-\int_{a}^{a+\omega} \dot{z}^{T}(s) \Theta \dot{z}(s) \mathrm{d} s \leq-\frac{2}{\omega}\left(\frac{1}{\omega} \int_{a}^{a+\omega} z(s) \mathrm{d} s-z(a)\right)^{T} \Theta\left(\frac{1}{\omega} \int_{a}^{a+\omega} z(s) \mathrm{d} s-z(a)\right) .
$$

Lemma 5 (see [34]). Assume that $\nu, \mu, \underline{\vartheta}, \bar{\vartheta}$ are real scalars such that $\nu \leq$ $1, \nu+\mu \leq 4$, and $\underline{\vartheta}<\bar{\vartheta}$. Let $\vartheta: \mathbb{R} \rightarrow(\underline{\vartheta}, \bar{\vartheta})$ be a real function. Then for any non-negative scalars $a, b$, the following inequality holds

$$
-\frac{a}{\vartheta(t)-\underline{\vartheta}}-\frac{b}{\bar{\vartheta}-\vartheta(t)} \leq \frac{1}{\bar{\vartheta}-\underline{\vartheta}} \max \{-\nu a-\mu b,-\mu a-\nu b\} .
$$

Remark 1. If we set $\nu=1, \mu=3$, then we get Lemma 3 of [33] from Lemma 5 . Thus, based on Lemma 5 , we can get some sufficient conditions of stochastic stability problem with less conservativeness.

\section{Main result}

As usual, a vector $x^{*}=\left(x_{1}^{*}, x_{2}^{*}, \ldots, x_{n}^{*}\right)^{T}$ is said to be an equilibrium point of system (1) if it satisfies

$$
\begin{aligned}
0= & -d_{i m} x_{i}{ }^{*}+\sum_{j=1}^{n} a_{i j m} f_{j}\left(x_{j}{ }^{*}\right)+\sum_{j=1}^{n} b_{i j m} f_{j}\left(x_{j}{ }^{*}\right)+\sum_{j=1}^{n} c_{i j} \varrho_{j}+\chi_{i} \\
& +\bigwedge_{j=1}^{n} \alpha_{i j} \int_{-\infty}^{t} k_{j}(t-s) f_{j}\left(x_{j}{ }^{*}\right) \mathrm{d} s+\bigvee_{j=1}^{n} \beta_{i j} \int_{-\infty}^{t} k_{j}(t-s) f_{j}\left(x_{j}{ }^{*}\right) \mathrm{d} s+ \\
& +\bigwedge_{j=1}^{n} \sigma_{i j} \varrho_{j}+\bigvee_{j=1}^{n} \delta_{i j} \varrho_{j} .
\end{aligned}
$$
$\mathbb{N}$.

Here, it is assumed that the impulse functions satisfy $J_{i k}\left(x_{i}^{*}\right)=0, k \in \mathbb{Z}_{+}, i \in$

In this paper, we always assume that some conditions are satisfied so that system (1) has a unique equilibrium point. To investigate the global asymptotic stability of the unique equilibrium point, we further assume that the impulsive function $J_{k}(\cdot)$ satisfies the following assumption

$$
J_{k}\left(x\left(t_{k}^{-}\right)\right)=-\Gamma_{k}\left\{x\left(t_{k}^{-}\right)-x^{*}\right\}, \quad \Gamma_{k}=\operatorname{diag}\left\{\gamma_{1 k}, \gamma_{2 k}, \cdots, \gamma_{n k}\right\}
$$


For the purpose of simplicity, we shift the intended equilibrium $x^{*}$ to the origin by letting $y_{i}(t)=x_{i}(t)-x_{i}^{*}$, and then the system (1) can be transformed into:

$$
\left\{\begin{aligned}
& \dot{y}_{i}(t)=- d_{i m} y_{i}(t)+\sum_{j=1}^{n} a_{i j m} g_{j}\left(y_{j}(t)\right)+\sum_{j=1}^{n} b_{i j m} g_{j}\left(y_{j}\left(t-\tau_{m}(t)\right)\right) \\
&+\bigwedge_{j=1}^{n} \alpha_{i j} \int_{-\infty}^{t} k_{j}(t-s) f_{j}\left(y_{j}(s)+x_{j}^{*}\right) \mathrm{d} s-\bigwedge_{j=1}^{n} \alpha_{i j} \int_{-\infty}^{t} k_{j}(t-s) f_{j}\left(x_{j}^{*}\right) \mathrm{d} s \\
&+\bigvee_{j=1}^{n} \beta_{i j} \int_{-\infty}^{t} k_{j}(t-s) f_{j}\left(y_{j}(s)+x_{j}^{*}\right) \mathrm{d} s-\bigvee_{j=1}^{n} \beta_{i j} \int_{-\infty}^{t} k_{j}(t-s) f_{j}\left(x_{j}^{*}\right) \mathrm{d} s \\
& t \neq t_{k}, \\
& \Delta y_{i}\left(t_{k}\right)=J_{i k}\left(y_{i}\left(t_{k}^{-}\right)\right)=-\gamma_{i k} y_{i}\left(t_{k}^{-}\right), \quad k \in \mathbb{Z}_{+}, \\
& y_{i}(s)=\psi_{i}(s):=\varphi_{i}(s)-x_{i}^{*}, \quad s \in(-\infty, 0]
\end{aligned}\right.
$$

where $g_{j}\left(y_{j}(\cdot)\right)=f_{j}\left(y_{j}(\cdot)+x_{j}^{*}\right)-f_{j}\left(x_{j}^{*}\right)$.

For convenience, we denote

$$
\begin{aligned}
& A_{m}=\left(a_{i j m}\right)_{n \times n}, B_{m}=\left(b_{i j m}\right)_{n \times n}, \\
& D_{m}=\operatorname{diag}\left\{d_{1 m}, d_{2 m}, \ldots, d_{n m}\right\}, y(t)=\left(y_{1}(t), y_{2}(t), \ldots, y_{n}(t)\right)^{T} .
\end{aligned}
$$

Before presenting the main results, for simplicity, we introduce a new vectors as

$$
\begin{aligned}
\zeta(t)=\operatorname{col}\left\{y(t), y\left(t-\tau_{m}(t)\right), y\left(t-\bar{\tau}_{m}\right), g(y(t)), g\left(y\left(t-\tau_{m}(t)\right)\right),\right. \\
\\
\left.\int_{-\infty}^{t} K(t-s) g(y(s)) \mathrm{d} s, \dot{y}(t), \zeta_{1}(t), \zeta_{2}(t)\right\},
\end{aligned}
$$

let $\varpi_{i}(i=1,2, \ldots, 9)$ be row vectors with block matrix entries, i.e., the $i$-th block is an identity matrix and the others are zero blocks, such that $y(t)=\varpi_{1} \zeta(t), y(t-$ $\left.\tau_{m}(t)\right)=\varpi_{2} \zeta(t)$, and so on.

Now for system (3), we give our main result about the stability of the equilibrium point.

Theorem 1 (See Appendix I for a proof). Assume that (H1)-(H5) hold. Then the unique equilibrium point of model (3) is globally asymptotically stable in mean square if there exist positive definite matrices $Q_{m}, R_{m}, S_{m}, W_{i}(i=1, \ldots, 5)$ and positive diagonal matrices $G, H, U, P_{m}, L_{m}, T_{m}, Z_{m}$ of appropriate dimensions such that

$$
\begin{aligned}
& \sum_{j=1}^{N} \pi_{m j}^{\prime}\left(Q_{j}+W_{5}\right)<W_{1} \\
& \sum_{j=1}^{N} \pi_{m j}^{\prime}\left(R_{j}\right)<W_{2}
\end{aligned}
$$


Zhang Y., Zheng C.-D.: Novel stochastic stability conditions of fuzzy...

$$
\begin{aligned}
& \sum_{j=1}^{N} \pi_{m j}^{\prime}\left(\bar{\tau}_{j} S_{j}\right)<W_{3}, \\
& {\left[\begin{array}{cc}
P_{m} & \left(I-\Gamma_{k}\right) P_{m} \\
* & P_{m}
\end{array}\right] \geq 0, \quad k \in \mathbb{Z}_{+},} \\
& \left(I-\Gamma_{k}\right) P_{m}\left(I-\Gamma_{k}\right) \leq P_{l}, \quad l \neq m, \quad l, m \in \mathcal{N}, \\
& {\left[\begin{array}{ccc}
\Phi_{m}-4 \mathcal{S}_{m}^{j}-\mathcal{W}_{\nu} & n \mathcal{P}_{m} & n \mathcal{Z}_{m} \\
* & -n H & 0 \\
* & 0 & -n G
\end{array}\right]<0, \quad j, \nu=1,2,}
\end{aligned}
$$

where

$$
\begin{aligned}
& \mathcal{S}_{m}^{1}=\left(\varpi_{8}-\varpi_{2}\right)^{T} S_{m}\left(\varpi_{8}-\varpi_{2}\right), \quad \mathcal{S}_{m}^{2}=\left(\varpi_{9}-\varpi_{3}\right)^{T} S_{m}\left(\varpi_{9}-\varpi_{3}\right), \\
& \mathcal{W}_{1}=-\left(\varpi_{1}-\varpi_{2}\right)^{T} W_{4}\left(\varpi_{1}-\varpi_{2}\right), \quad \mathcal{W}_{2}=-\left(\varpi_{2}-\varpi_{3}\right)^{T} W_{4}\left(\varpi_{2}-\varpi_{3}\right) \\
& \mathcal{P}_{m}=\operatorname{col}\left\{\begin{array}{lll}
P_{m} \Upsilon & 0_{8 n \times n}
\end{array}\right\}, \quad \mathcal{Z}_{m}=\operatorname{col}\left\{\begin{array}{lll}
0_{6 n \times n} & Z_{m} \Upsilon & 0_{2 n \times n}
\end{array}\right\}, \\
& \varpi_{i}=\left[\begin{array}{lll}
0_{(i-1) n \times n} & I & 0_{(9-i) n \times n}
\end{array}\right], i=1,2, \ldots, 9,
\end{aligned}
$$

with

$$
\begin{aligned}
& \Phi_{m}=\left[\Phi_{i j}\right]_{9 \times 9}, \\
& \Phi_{11}=-2 P_{m} D_{m}+Q_{m}+\bar{\tau} W_{1}-W_{4}+W_{5}-L_{m} \Lambda_{1}+\sum_{j=1}^{N} \pi_{m j} P_{j}, \\
& \Phi_{12}=W_{4}, \Phi_{14}=P_{m} A_{m}+L_{m} \Lambda_{2}, \Phi_{15}=P_{m} B_{m}, \Phi_{17}=-D_{m} Z_{m}, \\
& \Phi_{22}=-\left(1-\mu_{m}\right) Q_{m}-2 S_{m}-2 W_{4}-T_{m} \Lambda_{1}, \\
& \Phi_{23}=W_{4}, \Phi_{25}=T_{m} \Lambda_{2}, \Phi_{28}=2 S_{m}, \Phi_{33}=-2 S_{m}-W_{4}-W_{5}, \Phi_{39}=2 S_{m}, \\
& \Phi_{44}=R_{m}+\bar{\tau} W_{2}+U-L_{m}, \Phi_{47}=A_{m}^{T} Z_{m}, \Phi_{55}=-\left(1-\mu_{m}\right) R_{m}-T_{m}, \\
& \Phi_{57}=B_{m}^{T} Z_{m}, \Phi_{66}=H-U+G, \Phi_{77}=\bar{\tau}_{m}^{2} S_{m}+\bar{\tau}^{2}\left(\frac{1}{2} W_{3}+W_{4}\right)-2 Z_{m}, \\
& \Phi_{88}=\Phi_{99}=-2 S_{m},
\end{aligned}
$$

other parameters $\Phi_{i j}(1 \leq i<j \leq 9)$ are all equal to zeros, $\pi_{m j}^{\prime}=\max \left\{0, \pi_{m j}\right\}$ and

$$
\begin{array}{r}
\alpha=\left(\alpha_{i j}\right)_{n \times n}, \beta=\left(\beta_{i j}\right)_{n \times n},|\alpha|_{s}=\operatorname{diag}\left\{\sum_{i=1}^{n}\left|\alpha_{i 1}\right|, \sum_{i=1}^{n}\left|\alpha_{i 2}\right|, \ldots, \sum_{i=1}^{n}\left|\alpha_{i n}\right|\right\} \\
\Upsilon=|\alpha|_{s}+|\beta|_{s} .
\end{array}
$$

Remark 2. When $\dot{\tau}_{m}(t)$ is unknown or $\tau_{m}(t)$ is non-differentiable, we can verify the stability of model (3) by setting $Q_{m}=R_{m}=0$ in Theorem 1 .

Remark 3. Theorem 1 provides an LMI-based sufficient condition for the stability of the neural network (4). One advantage of the LMI approach is that the LMI condition can be checked numerically very efficiently by using the interiorpoint algorithms, which have been developed in solving LMIs by employing the 
Matlab LMI Toolbox. While the other conditions, which are based on the theory of M-matrix, matrix norm or algebraic inequality, ignore the signs of the weights, the neuron's excitatory and inhibitory effects, hence they result in conservativeness than LMI-based criteria.

If there is no Markovian jumping in system (3), similar to Theorem 1, we have the following result

Theorem 2 (See Appendix II for a proof). Assume that (H1)-(H5) hold. Then the unique equilibrium point of model (3) with $N=1$ is globally asymptotically stable if there exist positive definite matrices $Q, R, S, W_{4}, W_{5}$, positive diagonal matrices $E, G, H, U, P, L, T, Z$, any real matrix $X$ of appropriate dimensions such that

$$
\begin{aligned}
& {\left[\begin{array}{cc}
Q & X \\
* & R
\end{array}\right]>0,} \\
& {\left[\begin{array}{cc}
P & \left(I-\Gamma_{k}\right) P \\
* & P
\end{array}\right] \geq 0, \quad k \in \mathbb{Z}_{+},} \\
& {\left[\begin{array}{ccc}
\Phi-4 \mathcal{S}_{j}-\mathcal{W}_{\nu} & n \mathcal{P} & n \mathcal{Z} \\
* & -n H & 0 \\
* & 0 & -n G
\end{array}\right]<0, \quad j, \nu=1,2,}
\end{aligned}
$$

where

$$
\begin{aligned}
\mathcal{S}_{1} & =\left(\varpi_{8}-\varpi_{2}\right)^{T} S\left(\varpi_{8}-\varpi_{2}\right), \quad \mathcal{S}_{2}=\left(\varpi_{9}-\varpi_{3}\right)^{T} S\left(\varpi_{9}-\varpi_{3}\right), \\
\mathcal{W}_{1} & =-\left(\varpi_{1}-\varpi_{2}\right)^{T} W_{4}\left(\varpi_{1}-\varpi_{2}\right), \quad \mathcal{W}_{2}=-\left(\varpi_{2}-\varpi_{3}\right)^{T} W_{4}\left(\varpi_{2}-\varpi_{3}\right) \\
\mathcal{P} & =\operatorname{col}\left\{\begin{array}{lllll}
P \Upsilon & 0_{8 n \times n}
\end{array}\right\}, \quad \mathcal{Z}=\operatorname{col}\left\{\begin{array}{lll}
0_{6 n \times n} & Z \Upsilon & 0_{2 n \times n}
\end{array}\right\},
\end{aligned}
$$

with

$$
\begin{aligned}
& \Phi=\left[\Phi_{i j}\right]_{9 \times 9}, \\
& \Phi_{11}=\Lambda_{4} E \Lambda_{4}+\Lambda_{3} R \Lambda_{3}-2 P D+Q-W_{4}+W_{5}-L \Lambda_{1}-X \Lambda_{3}-\Lambda_{3} X^{T}, \\
& \Phi_{12}=W_{4}, \Phi_{14}=P A+L \Lambda_{2}+X-\Lambda_{3} R, \Phi_{15}=P B, \Phi_{17}=-D Z \\
& \Phi_{22}=-(1-\mu)\left(Q+\Lambda_{4} E \Lambda_{4}+\Lambda_{3} R \Lambda_{3}-X \Lambda_{3}-\Lambda_{3} X^{T}\right)-2 S-2 W_{4}-T \Lambda_{1}, \\
& \Phi_{23}=W_{4}, \Phi_{25}=T \Lambda_{2}-(1-\mu)\left(X-\Lambda_{3} R\right), \Phi_{28}=2 S, \Phi_{33}=-2 S-W_{4}-W_{5}, \\
& \Phi_{39}=2 S, \Phi_{44}=R+U-L-E, \Phi_{47}=A^{T} Z, \Phi_{55}=-(1-\mu)(R-E)-T, \\
& \Phi_{57}=B^{T} Z, \Phi_{66}=H-U+G, \Phi_{77}=\bar{\tau}^{2}\left(S+W_{4}\right)-2 Z, \Phi_{88}=\Phi_{99}=-2 S .
\end{aligned}
$$

Remark 4. When $\dot{\tau}(t)$ is unknown or $\tau(t)$ is non-differentiable, we can verify the stability of model (3) with $N=1$ by setting $Q=R=0$ in Theorem 2 .

\section{Illustrative examples}

In this section, we provide two numerical examples to demonstrate the effectiveness of our delay-dependent stability criteria. 
Example 1. Consider system (3) with $n=N=2$ and

$$
\begin{aligned}
& D_{1}=\left[\begin{array}{cc}
3.1 & 0 \\
0 & 3.4
\end{array}\right], D_{2}=\left[\begin{array}{cc}
3.0 & 0 \\
0 & 3.3
\end{array}\right], A_{1}=\left[\begin{array}{cc}
1.1 & -0.7 \\
0.9 & 1.2
\end{array}\right], \\
& A_{2}=\left[\begin{array}{cc}
-0.8 & -1.1 \\
0.9 & 0.8
\end{array}\right], \quad B_{1}=\left[\begin{array}{cc}
1.2 & 0.6 \\
0.8 & 1
\end{array}\right], \quad B_{2}=\left[\begin{array}{cc}
-0.6 & -0.7 \\
0.7 & 0.6
\end{array}\right], \\
& \alpha=\frac{1}{32}\left[\begin{array}{cc}
1 & -1 \\
1 & 1
\end{array}\right], \quad \beta=\frac{1}{32}\left[\begin{array}{cc}
1 & 1 \\
-1 & 1
\end{array}\right], \quad \tau_{1}(t)=0.2+0.2 \sin (t), \\
& \tau_{2}(t)=0.29+0.15 \cos (2 t), f_{i}(x)=\tanh (x), k_{i}(s)=\exp (-s), i=1,2 .
\end{aligned}
$$

It is easy to see that assumptions (H1), (H2) are satisfied with $\bar{\tau}_{1}=0.4, \bar{\tau}=\bar{\tau}_{2}=$ $0.44, \tau_{1}^{\prime}=0.2, \tau_{2}^{\prime}=0.3, \Lambda_{1}=0, \Lambda_{2}=0.5 I$. Furthermore, we set $t_{k}=0.4 k$ and $\Gamma_{k}=0.4 I$ in assumptions (H4), (H5), $k \in \mathbb{Z}_{+}$, then assumption (H3) is satisfied. In addition, we set $\pi_{11}=-0.6, \pi_{12}=0.6, \pi_{21}=0.4, \pi_{22}=-0.4$, by using the Matlab LMI Toolbox, the LMIs (4)-(9) are feasible. Thus the unique equilibrium point of model (3) is globally asymptotically stable. One solution is as follows:

$$
\begin{aligned}
& P_{1}=\operatorname{diag}\{0.7598,0.1359\}, P_{2}=\operatorname{diag}\{0.2745,0.1344\}, \\
& Q_{1}=\left(\begin{array}{cc}
0.0259 & -0.0056 \\
-0.0056 & 0.0077
\end{array}\right), Q_{2}=\left(\begin{array}{cc}
0.0218 & -0.0026 \\
-0.0026 & 0.0049
\end{array}\right) \text {, } \\
& R_{1}=\left(\begin{array}{ll}
0.7583 & 0.3465 \\
0.3465 & 0.1612
\end{array}\right), R_{2}=\left(\begin{array}{ll}
0.7678 & 0.3408 \\
0.3408 & 0.1629
\end{array}\right) \text {, } \\
& S_{1}=\left(\begin{array}{cc}
0.0492 & -0.0114 \\
-0.0114 & 0.0094
\end{array}\right), S_{2}=\left(\begin{array}{cc}
0.0201 & -0.0141 \\
-0.0141 & 0.0178
\end{array}\right) \text {, } \\
& W_{1}=\left(\begin{array}{cc}
0.0058 & -0.0028 \\
-0.0028 & 0.0024
\end{array}\right), W_{2}=\left(\begin{array}{cc}
0.0090 & -0.0043 \\
-0.0043 & 0.0033
\end{array}\right) \text {, } \\
& W_{3}=\left(\begin{array}{cc}
0.0146 & -0.0066 \\
-0.0066 & 0.0060
\end{array}\right), W_{4}=\left(\begin{array}{cc}
0.0063 & -0.0028 \\
-0.0028 & 0.0025
\end{array}\right) \text {, } \\
& W_{4}=\left(\begin{array}{ll}
0.1115 & 0.0303 \\
0.0303 & 0.1397
\end{array}\right), \quad U=\operatorname{diag}\{0.1466,0.0338\}, \\
& G=\operatorname{diag}\{0.0098,0.0079\}, \quad H=\operatorname{diag}\{0.1340,0.0248\} \text {, } \\
& L_{1}=\operatorname{diag}\{4.7068,0.5806\}, \quad L_{2}=\operatorname{diag}\{3.3288,0.7443\} \text {, } \\
& T_{1}=\operatorname{diag}\{0.2415,0.2287\}, T_{2}=\operatorname{diag}\{0.1233,0.0903\} \text {, } \\
& Z_{1}=\operatorname{diag}\{0.0466,0.0307\}, \quad Z_{2}=\operatorname{diag}\{0.0308,0.0341\} .
\end{aligned}
$$

For numerical simulation, we consider the initial state $(0.4,-0.4)^{T}$ in mode 1 and $(-0.4,0.4)^{T}$ in mode 2. Fig. 1 and Fig. 2 depict the time responses of state variables $y_{1}(t), y_{2}(t)$ in mode 1 and mode 2 with step 0.01 respectively. It confirms that the proposed condition in Theorem 1 leads to globally asymptotic stable equilibrium point for the model (3).

However, it is verified that the LMIs of Theorem 1 in [1] admit no feasible solutions, that is, for this example, the condition of Ref. [1] fails to assure the stability. Therefore, we can say that for this system, the results in this paper are much effective and less conservative than those in [1]. 
Neural Network World 1/2016, 7-28

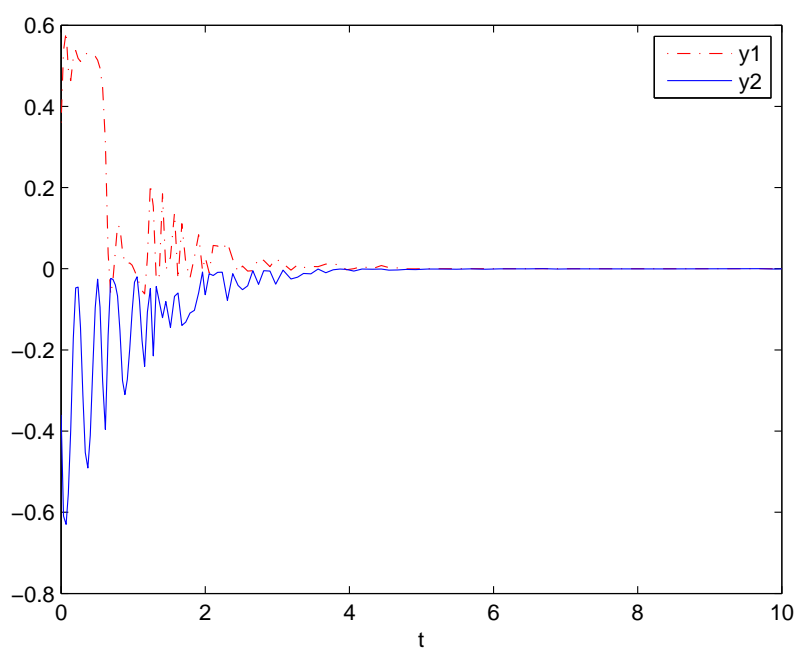

Fig. 1 The state trajectory of the mode 1 with initial value $(0.4,-0.4)^{T}$ in Example 1 .

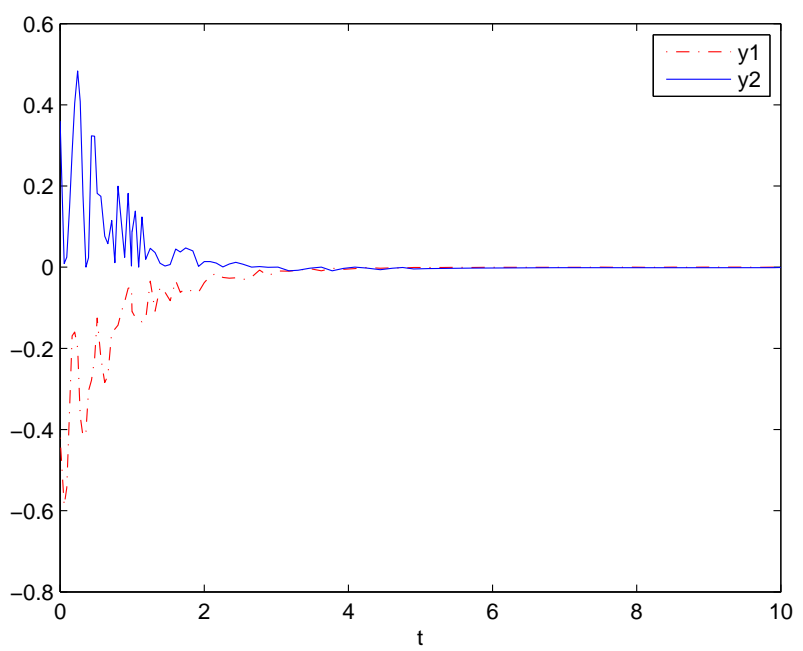

Fig. 2 The state trajectory of the mode 2 with initial value $(-0.4,0.4)^{T}$ in Example 1 .

Example 2. Consider system (3) with $n=2, N=1$ and

$$
\begin{aligned}
& D=\left[\begin{array}{cc}
2.7 & 0 \\
0 & 2.5
\end{array}\right], \quad A=\left[\begin{array}{cc}
1.8 & -0.9 \\
1.5 & -0.8
\end{array}\right], \quad B=\left[\begin{array}{cc}
-1.4 & 0.6 \\
2.5 & -0.8
\end{array}\right], \\
& \alpha=\frac{1}{16}\left[\begin{array}{cc}
1 & 1 \\
1 & -1
\end{array}\right], \quad \beta=\frac{1}{16}\left[\begin{array}{cc}
1 & -1 \\
-1 & 1
\end{array}\right], \quad \tau(t)=0.2+0.2 \sin (2 t), \\
& f_{i}(x)=\frac{1}{2}(|x+1|-|x-1|), k_{i}(s)=\frac{2}{\pi} \frac{1}{1+s^{2}}, i=1,2 .
\end{aligned}
$$


It is easy to see that assumptions (H1) and (H2) are satisfied with $\tau=0.4, \tau^{\prime}=$ $0.4, \Lambda_{1}=0, \Lambda_{2}=0.5 I$. Furthermore, we set $t_{k}=0.5 k$ and $\Gamma_{k}=0.4 I$ in assumptions (H4),(H5), $k \in \mathbb{Z}_{+}$, then assumption (H3) is satisfied. By utilizing the Matlab LMI Toolbox, the LMIs (11)-(12) are feasible. Thus from Theorem 2 we conclude that the unique equilibrium point of model (3) is globally asymptotically stable.

However, it is easy to see that none of the conditions in $[5,11]$ can be applied to verify the stability of this model. Therefore, we can say that for this system of Example 2, the results in this paper are much effective and less conservative than those in $[5,11]$.

\section{Conclusions}

This paper investigates the stochastic stability of fuzzy neural networks with Markovian jumping parameters and mixed delays under impulsive perturbations in mean square. The mixed delays consist of time-varying delay and continuously distributed delay. By employing a new Lyapunov-Krasovskii functional, linear convex combination technique, a novel reciprocal convex lemma and the free-weight matrix method, two novel sufficient conditions are derived to ensure the stochastic asymptotic stability of the equilibrium point of the considered networks in mean square. The proposed results, which are expressed in terms of linear matrix inequalities, can be easily checked via Matlab LMI Toolbox. One of the future research topics would be an extension of the present results to other systems, for example, synchronization of Markovian coupled neural networks, stochastic fuzzy Markovian jumping neural networks with leakage delay under impulsive perturbations, etc.

\section{Appendix I Proof of Theorem 1}

Construct a Lyapunov-Krasovskii functional in the following form

$$
V_{m}\left(t, y_{t}\right)=\sum_{i=1}^{6} V_{i m}\left(t, y_{t}\right),
$$

where

$$
\begin{aligned}
V_{1 m}\left(t, y_{t}\right)= & y(t)^{T} P_{m} y(t) \\
V_{2 m}\left(t, y_{t}\right)= & \int_{t-\tau_{m}(t)}^{t}\left[y(s)^{T} Q_{m} y(s)+g(y(s))^{T} R_{m} g(y(s))\right] \mathrm{d} s, \\
V_{3 m}\left(t, y_{t}\right)= & \bar{\tau}_{m} \int_{t-\bar{\tau}_{m}}^{t} \int_{\theta}^{t} \dot{y}(s)^{T} S_{m} \dot{y}(s) \mathrm{d} s \mathrm{~d} \theta, \\
V_{4 m}\left(t, y_{t}\right)= & \int_{t-\bar{\tau}}^{t} \int_{\theta}^{t}\left[y(s)^{T} W_{1} y(s)+g(y(s))^{T} W_{2} g(y(s))\right] \mathrm{d} s \mathrm{~d} \theta \\
& +\int_{t-\bar{\tau}}^{t} \int_{\theta}^{t} \int_{\eta}^{t} \dot{y}(s)^{T} W_{3} \dot{y}(s) \mathrm{d} s \mathrm{~d} \eta \mathrm{d} \theta,
\end{aligned}
$$




\section{Neural Network World 1/2016, 7-28}

$$
\begin{aligned}
& V_{5 m}\left(t, y_{t}\right)=\bar{\tau} \int_{t-\bar{\tau}}^{t} \int_{\theta}^{t} \dot{y}(s)^{T} W_{4} \dot{y}(s) \mathrm{d} s \mathrm{~d} \theta+\int_{t-\bar{\tau}_{m}}^{t} y(s)^{T} W_{5} y(s) \mathrm{d} s, \\
& V_{6 m}\left(t, y_{t}\right)=\sum_{j=1}^{n} u_{j} \int_{0}^{\infty} k_{j}(\theta) \int_{t-\theta}^{t} g_{j}^{2}\left(y_{j}(s)\right) \mathrm{d} s \mathrm{~d} \theta .
\end{aligned}
$$

Denoting by $\mathfrak{L}$ the weak infinitesimal generator of the random process $\{y(t)$, $\eta(t)\}_{t \geq 0}$

$$
\begin{gathered}
\mathfrak{L} V\left(y_{t}, t, m\right):=\lim _{\delta \rightarrow 0^{+}} \frac{1}{\delta} \sup \left[\mathbb{E}\left\{V\left(y_{t+\delta}, t+\delta, \eta(t+\delta)\right) \mid y_{t}, \eta(t)=m\right\}-\right. \\
\left.-V\left(y_{t}, t, \eta(t)=m\right)\right],
\end{gathered}
$$

calculating the weak infinitesimal operator along the system (3) gives

$$
\mathfrak{L} V_{m}\left(t, y_{t}\right)=\sum_{i=1}^{6} \mathfrak{L} V_{i m}\left(t, y_{t}\right),
$$

where

$$
\begin{aligned}
\mathfrak{L} V_{1 m}\left(t, y_{t}\right)= & 2 y(t)^{T} P_{m} \dot{y}(t)+\sum_{j=1}^{N} \pi_{m j} y(t)^{T} P_{j} y(t) \\
= & 2 y(t)^{T} P_{m}\left\{-D_{m} y(t)+A_{m} g(y(t))+B_{m} g\left(y\left(t-\tau_{m}(t)\right)\right)\right\}+ \\
& \quad+\sum_{j=1}^{N} \pi_{m j} y(t)^{T} P_{j} y(t) \\
+ & 2 \sum_{i=1}^{n} p_{i m} y_{i}(t)\left(\bigwedge_{j=1}^{n} \alpha_{i j} \int_{-\infty}^{t} k_{j}(t-s) f_{j}\left(y_{j}(s)+x_{j}^{*}\right) \mathrm{d} s\right. \\
- & \bigwedge_{j=1}^{n} \alpha_{i j} f_{j}\left(x_{j}^{*}\right)+\bigvee_{j=1}^{n} \beta_{i j} \int_{-\infty}^{t} k_{j}(t-s) f_{j}\left(y_{j}(s)+x_{j}^{*}\right) \mathrm{d} s- \\
& \left.\quad-\bigvee_{j=1}^{n} \beta_{i j} f_{j}\left(x_{j}^{*}\right)\right),
\end{aligned}
$$

$$
\begin{aligned}
\mathfrak{L} V_{2 m}\left(t, y_{t}\right)= & y(t)^{T} Q_{m} y(t)-\left(1-\dot{\tau}_{m}(t)\right) y\left(t-\tau_{m}(t)\right)^{T} Q_{m} y\left(t-\tau_{m}(t)\right) \\
& +g(y(t))^{T} R_{m} g(y(t))-\left(1-\dot{\tau}_{m}(t)\right) g\left(y\left(t-\tau_{m}(t)\right)\right)^{T} R_{m} g\left(y\left(t-\tau_{m}(t)\right)\right) \\
& +\sum_{j=1}^{N} \pi_{m j} \int_{t-\tau_{j}(t)}^{t}\left[y(s)^{T} Q_{j} y(s)+g(y(s))^{T} R_{j} g(y(s))\right] \mathrm{d} s \\
\leq & y(t)^{T} Q_{m} y(t)-\left(1-\mu_{m}\right) y\left(t-\tau_{m}(t)\right)^{T} Q_{m} y\left(t-\tau_{m}(t)\right) \\
& +g(y(t))^{T} R_{m} g(y(t))-\left(1-\mu_{m}\right) g\left(y\left(t-\tau_{m}(t)\right)\right)^{T} R_{m} g\left(y\left(t-\tau_{m}(t)\right)\right) \\
& +\sum_{j=1}^{N} \pi_{m j}^{\prime} \int_{t-\bar{\tau}}^{t}\left[y(s)^{T}\left(Q_{j}\right) y(s)+g(y(s))^{T}\left(R_{j}\right) g(y(s))\right] \mathrm{d} s
\end{aligned}
$$




$$
\begin{aligned}
& \mathfrak{L} V_{3 m}\left(t, y_{t}\right)=\bar{\tau}_{m}^{2} \dot{y}(t)^{T} S_{m} \dot{y}(t)+\sum_{j=1}^{N} \pi_{m j} \bar{\tau}_{j} \int_{t-\bar{\tau}_{j}}^{t} \int_{\theta}^{t} \dot{y}(s)^{T} S_{j} \dot{y}(s) \mathrm{d} s \mathrm{~d} \theta \\
& -\bar{\tau}_{m} \int_{t-\bar{\tau}_{m}}^{t} \dot{y}(s)^{T} S_{m} \dot{y}(s) \mathrm{d} s \\
& \leq \bar{\tau}_{m}^{2} \dot{y}(t)^{T} S_{m} \dot{y}(t)-\bar{\tau}_{m} \int_{t-\bar{\tau}_{m}}^{t} \dot{y}(s)^{T} S_{m} \dot{y}(s) \mathrm{d} s \\
& +\sum_{j=1}^{N} \pi_{m j}^{\prime} \int_{t-\bar{\tau}}^{t} \int_{\theta}^{t} \dot{y}(s)^{T}\left(\bar{\tau}_{j} S_{j}\right) \dot{y}(s) \mathrm{d} s \mathrm{~d} \theta \\
& \mathfrak{L} V_{4 m}\left(t, y_{t}\right)=\bar{\tau}\left[y(t)^{T} W_{1} y(t)+g(y(t))^{T} W_{2} g(y(t))\right] \\
& -\int_{t-\bar{\tau}}^{t}\left[y(s)^{T} W_{1} y(s)+g(y(s))^{T} W_{2} g(y(s))\right] \mathrm{d} s \\
& +\frac{\bar{\tau}^{2}}{2} \dot{y}(t)^{T} W_{3} \dot{y}(t)-\int_{t-\bar{\tau}}^{t} \int_{\theta}^{t} \dot{y}(s)^{T} W_{3} \dot{y}(s) \mathrm{d} s \mathrm{~d} \theta, \\
& \mathfrak{L} V_{5 m}\left(t, y_{t}\right)=\bar{\tau}^{2} \dot{y}(t)^{T} W_{4} \dot{y}(t)-\bar{\tau} \int_{t-\bar{\tau}}^{t} \dot{y}(s)^{T} W_{4} \dot{y}(s) \mathrm{d} s+y(t)^{T} W_{5} y(t) \\
& -y\left(t-\bar{\tau}_{m}\right)^{T} W_{5} y\left(t-\bar{\tau}_{m}\right)+\sum_{j=1}^{N} \pi_{m j} \int_{t-\tau_{j}(t)}^{t} y(s)^{T} W_{5} y(s) \mathrm{d} s \\
& \leq \bar{\tau}^{2} \dot{y}(t)^{T} W_{4} \dot{y}(t)-\bar{\tau}_{m} \int_{t-\bar{\tau}_{m}}^{t} \dot{y}(s)^{T} W_{4} \dot{y}(s) \mathrm{d} s+y(t)^{T} W_{5} y(t) \\
& -y\left(t-\bar{\tau}_{m}\right)^{T} W_{5} y\left(t-\bar{\tau}_{m}\right)+\sum_{j=1}^{N} \pi_{m j}^{\prime} \int_{t-\bar{\tau}}^{t} y(s)^{T} W_{5} y(s) \mathrm{d} s, \\
& \mathfrak{L} V_{6 m}\left(t, y_{t}\right)=\sum_{j=1}^{n} u_{j} \int_{0}^{\infty} k_{j}(\theta) g_{j}^{2}\left(y_{j}(t)\right) \mathrm{d} \theta-\sum_{j=1}^{n} u_{j} \int_{0}^{\infty} k_{j}(\theta) g_{j}^{2}\left(y_{j}(t-\theta)\right) \mathrm{d} \theta
\end{aligned}
$$

with $P_{m}=\operatorname{diag}\left\{p_{1 m}, p_{2 m}, \ldots, p_{n m}\right\}$.

In order to get stability result expressed in terms of linear matrix inequalities, we need to handle the terms with fuzzy logic in $\mathfrak{L} V_{1 m}(t, y(t))$. Based on Lemma 2, the following inequalities hold

$$
\begin{aligned}
& \left|\bigwedge_{j=1}^{n} \alpha_{i j} \int_{-\infty}^{t} k_{j}(t-s) f_{j}\left(y_{j}(s)+x_{j}^{*}\right) \mathrm{d} s-\bigwedge_{j=1}^{n} \alpha_{i j} \int_{-\infty}^{t} k_{j}(t-s) f_{j}\left(x_{j}^{*}\right) \mathrm{d} s\right| \\
\leq & \sum_{j=1}^{n}\left|\alpha_{i j}\right| \times\left|\int_{-\infty}^{t} k_{j}(t-s) f_{j}\left(y_{j}(s)+x_{j}^{*}\right) \mathrm{d} s-\int_{-\infty}^{t} k_{j}(t-s) f_{j}\left(x_{j}^{*}\right) \mathrm{d} s\right|
\end{aligned}
$$




$$
\begin{aligned}
= & \sum_{j=1}^{n}\left|\alpha_{i j}\right| \times\left|\int_{-\infty}^{t} k_{j}(t-s) g_{j}\left(y_{j}(s)\right) \mathrm{d} s\right| \\
& \left|\bigvee_{j=1}^{n} \beta_{i j} \int_{-\infty}^{t} k_{j}(t-s) f_{j}\left(y_{j}(s)+x_{j}^{*}\right) \mathrm{d} s-\bigvee_{j=1}^{n} \beta_{i j} \int_{-\infty}^{t} k_{j}(t-s) f_{j}\left(x_{j}^{*}\right) \mathrm{d} s\right| \\
\leq & \sum_{j=1}^{n}\left|\beta_{i j}\right| \times\left|\int_{-\infty}^{t} k_{j}(t-s) f_{j}\left(y_{j}(s)+x_{j}^{*}\right) \mathrm{d} s-\int_{-\infty}^{t} k_{j}(t-s) f_{j}\left(x_{j}^{*}\right) \mathrm{d} s\right| \\
= & \sum_{j=1}^{n}\left|\beta_{i j}\right| \times\left|\int_{-\infty}^{t} k_{j}(t-s) g_{j}\left(y_{j}(s)\right) \mathrm{d} s\right| .
\end{aligned}
$$

Lemmas 1 and 3 play an important role in establishing LMI-based stability conditions for fuzzy neural networks. By applying Lemmas 1 and 3, we obtain the following inequalities for any positive diagonal matrix $H$

$$
\begin{aligned}
& 2 \sum_{i=1}^{n} p_{i m} y_{i}(t)\left(\bigwedge_{j=1}^{n} \alpha_{i j} \int_{-\infty}^{t} k_{j}(t-s) f_{j}\left(y_{j}(s)+x_{j}^{*}\right) \mathrm{d} s-\right. \\
& \quad-\bigwedge_{j=1}^{n} \alpha_{i j} \int_{-\infty}^{t} k_{j}(t-s) f_{j}\left(x_{j}^{*}\right) \mathrm{d} s+\bigvee_{j=1}^{n} \beta_{i j} \int_{-\infty}^{t} k_{j}(t-s) f_{j}\left(y_{j}(s)+x_{j}^{*}\right) \mathrm{d} s- \\
& \left.\quad-\bigvee_{j=1}^{n} \beta_{i j} \int_{-\infty}^{t} k_{j}(t-s) f_{j}\left(x_{j}^{*}\right) \mathrm{d} s\right) \\
& \leq 2 \sum_{i=1}^{n} p_{i m}\left|y_{i}(t)\right|\left(\mid \bigwedge_{j=1}^{n} \alpha_{i j} \int_{-\infty}^{t} k_{j}(t-s) f_{j}\left(y_{j}(s)+x_{j}^{*}\right) \mathrm{d} s-\right. \\
& \quad-\bigwedge_{j=1}^{n} \alpha_{i j} \int_{-\infty}^{t} k_{j}(t-s) f_{j}\left(x_{j}^{*}\right) \mathrm{d} s|+| \bigvee_{j=1}^{n} \beta_{i j} \int_{-\infty}^{t} k_{j}(t-s) f_{j}\left(y_{j}(s)+x_{j}^{*}\right) \mathrm{d} s- \\
& \left.\bigvee_{j=1}^{n} \beta_{i j} \int_{-\infty}^{t} k_{j}(t-s) f_{j}\left(x_{j}^{*}\right) \mathrm{d} s \mid\right) \\
& \leq 2|y(t)|^{T} P_{m}(|\alpha|+|\beta|)\left|\int_{-\infty}^{t} k(t-s) g(y(s)) \mathrm{d} s\right| \\
& \leq|y(t)|^{T} P_{m}(|\alpha|+|\beta|) H^{-1}(|\alpha|+|\beta|) P_{m}|y(t)| \\
& +\left|\int_{-\infty}^{t} k(t-s) g(y(s)) \mathrm{d} s\right| H_{-\infty}^{T} k(t-s) g(y(s)) \mathrm{d} s \mid \\
& \leq n y(t)^{T} P_{m} \Upsilon H^{-1} \Upsilon P_{m} y(t)+\left(\int_{-\infty}^{t} k(t-s) g(y(s)) \mathrm{d} s\right)^{T} \\
& H\left(\int_{-\infty}^{t} k(t-s) g(y(s)) \mathrm{d} s\right)
\end{aligned}
$$

Lemmas 1, 4 and the Leibniz-Newton formulae are important tools for our obtaining less conservative stability criteria by means of linear matrix inequalities. 
For $0<\tau_{m}(t) \leq \bar{\tau}_{m}$, define $\zeta_{1}(t)=\frac{1}{\tau_{m}(t)} \int_{t-\tau_{m}(t)}^{t} y(s) \mathrm{d} s$. It is easy to see that $\zeta_{1}(t) \rightarrow y(t)$ while $\tau_{m}(t) \rightarrow 0$. Therefore we can define $\zeta_{1}(t)=y(t)$ when $\tau_{m}(t)=0$. Similarly, for $0 \leq \tau_{m}(t)<\bar{\tau}_{m}$, define $\zeta_{2}(t)=\frac{1}{\bar{\tau}_{m}-\tau_{m}(t)} \int_{t-\bar{\tau}_{m}}^{t-\tau_{m}(t)} y(s) \mathrm{d} s ;$ when $\tau_{m}(t)=\bar{\tau}_{m}$, define $\zeta_{2}(t)=y\left(t-\bar{\tau}_{m}\right)$.

For $0<\tau_{m}(t)<\bar{\tau}_{m}$, utilizing Lemma 4 gives

$$
\begin{aligned}
& -\int_{t-\bar{\tau}_{m}}^{t} \dot{y}(s)^{T} S_{m} \dot{y}(s) \mathrm{d} s \\
= & -\int_{t-\tau_{m}(t)}^{t} \dot{y}(s)^{T} S_{m} \dot{y}(s) \mathrm{d} s-\int_{t-\bar{\tau}_{m}}^{t-\tau_{m}(t)} \dot{y}(s)^{T} S_{m} \dot{y}(s) \mathrm{d} s \\
\leq & -\frac{2}{\tau_{m}(t)}\left[\zeta_{1}(t)-y\left(t-\tau_{m}(t)\right)\right]^{T} S_{m}\left[\zeta_{1}(t)-y\left(t-\tau_{m}(t)\right)\right] \\
& -\frac{2}{\bar{\tau}_{m}-\tau_{m}(t)}\left[\zeta_{2}(t)-y\left(t-\bar{\tau}_{m}\right)\right]^{T} S_{m}\left[\zeta_{2}(t)-y\left(t-\bar{\tau}_{m}\right)\right] .
\end{aligned}
$$

Defining

$$
\begin{aligned}
& \Xi_{1}=\left[\zeta_{1}(t)-y\left(t-\tau_{m}(t)\right)\right]^{T} S_{m}\left[\zeta_{1}(t)-y\left(t-\tau_{m}(t)\right)\right], \\
& \Xi_{2}=\left[\zeta_{2}(t)-y\left(t-\bar{\tau}_{m}\right)\right]^{T} S_{m}\left[\zeta_{2}(t)-y\left(t-\bar{\tau}_{m}\right)\right],
\end{aligned}
$$

we get the following inequality from inequality (21) and Lemma 5 with $\nu=1, \mu=3$

$$
-\bar{\tau}_{m} \int_{t-\bar{\tau}_{m}}^{t} \dot{y}(s)^{T} S_{m} \dot{y}(s) \mathrm{d} s \leq 2 \max \left\{-\Xi_{1}-3 \Xi_{2},-3 \Xi_{1}-\Xi_{2}\right\} .
$$

It is easy to see that inequality (22) holds for any $t>0$ with $\tau_{m}(t)=0$ or $\tau_{m}(t)=\bar{\tau}_{m}$.

On the other hand, by using the well-known Jensen integral inequality and the Leibniz-Newton formulae, we derive

$$
\begin{aligned}
& -\bar{\tau}_{m} \int_{t-\bar{\tau}_{m}}^{t} \dot{y}(s)^{T} W_{4} \dot{y}(s) \mathrm{d} s \\
= & -\bar{\tau}_{m} \int_{t-\tau_{m}(t)}^{t} \dot{y}(s)^{T} W_{4} \dot{y}(s) \mathrm{d} s-\bar{\tau}_{m} \int_{t-\bar{\tau}_{m}}^{t-\tau_{m}(t)} \dot{y}(s)^{T} W_{4} \dot{y}(s) \mathrm{d} s \\
= & -\left[\tau_{m}(t)+\left(\bar{\tau}_{m}-\tau_{m}(t)\right)\right] \int_{t-\tau_{m}(t)}^{t} \dot{y}(s)^{T} W_{4} \dot{y}(s) \mathrm{d} s \\
& -\left[\left(\bar{\tau}_{m}-\tau_{m}(t)\right)+\tau_{m}(t)\right] \int_{t-\bar{\tau}_{m}}^{t-\tau_{m}(t)} \dot{y}(s)^{T} W_{4} \dot{y}(s) \mathrm{d} s . \\
\leq & -\left[1+\frac{\bar{\tau}_{m}-\tau_{m}(t)}{\bar{\tau}_{m}}\right] \tau_{m}(t) \int_{t-\tau_{m}(t)}^{t} \dot{y}(s)^{T} W_{4} \dot{y}(s) \mathrm{d} s
\end{aligned}
$$




$$
\begin{aligned}
& -\left[1+\frac{\tau_{m}(t)}{\bar{\tau}_{m}}\right]\left(\bar{\tau}_{m}-\tau_{m}(t)\right) \int_{t-\tau_{m}}^{t-\tau_{m}(t)} \dot{y}(s)^{T} W_{4} \dot{y}(s) \mathrm{d} s \\
\leq & -\left[1+\frac{\bar{\tau}_{m}-\tau_{m}(t)}{\bar{\tau}_{m}}\right]\left(\int_{t-\tau_{m}(t)}^{t} \dot{y}(s) \mathrm{d} s\right)^{T} W_{4}\left(\int_{t-\tau_{m}(t)}^{t} \dot{y}(s) \mathrm{d} s\right) \\
- & {\left[1+\frac{\tau_{m}(t)}{\bar{\tau}_{m}}\right]\left(\int_{t-\bar{\tau}_{m}}^{t-\tau_{m}(t)} \dot{y}(s) \mathrm{d} s\right)^{T} W_{4}\left(\int_{t-\bar{\tau}_{m}}^{t-\tau_{m}(t)} \dot{y}(s) \mathrm{d} s\right) } \\
= & \zeta(t)^{T}\left\{\left[1+\frac{\bar{\tau}_{m}-\tau_{m}(t)}{\bar{\tau}_{m}}\right]\left(\varpi_{1}-\varpi_{2}\right)^{T} W_{4}\left(\varpi_{1}-\varpi_{2}\right)\right. \\
& \left.+\left[1+\frac{\tau_{m}(t)}{\bar{\tau}_{m}}\right]\left(\varpi_{2}-\varpi_{3}\right)^{T} W_{4}\left(\varpi_{2}-\varpi_{3}\right)\right\} \zeta(t) .
\end{aligned}
$$

Cauchy-Schwarz inequality is an effective tool for neural networks with unbounded continuously distributed delay to derive LMI-based stability condition. From Eq. (2) and Cauchy-Schwarz inequality, we get the following inequality

$$
\begin{aligned}
& \mathfrak{L} V_{6 m}\left(t, y_{t}\right)=g(y(t))^{T} U g(y(t))-\sum_{j=1}^{n} u_{j} \int_{0}^{\infty} k_{j}(\theta) \mathrm{d} \theta \int_{0}^{\infty} k_{j}(\theta) g_{j}^{2}\left(y_{j}(t-\theta)\right) \mathrm{d} \theta \\
& \leq g(y(t))^{T} U g(y(t))-\sum_{j=1}^{n} u_{j}\left(\int_{0}^{\infty} k_{j}(\theta) g_{j}\left(y_{j}(t-\theta)\right) \mathrm{d} \theta\right)^{2} \\
&= g(y(t))^{T} U g(y(t))-\left(\int_{-\infty}^{t} K(t-s) g(y(s)) \mathrm{d} s\right)^{T} \\
& U\left(\int_{-\infty}^{t} K(t-s) g(y(s)) \mathrm{d} s\right)
\end{aligned}
$$

with $U=\operatorname{diag}\left\{u_{1}, u_{2}, \ldots, u_{n}\right\}, K(t-s)=\operatorname{diag}\left\{k_{1}(t-s), k_{2}(t-s), \ldots, k_{n}(t-s)\right\}$.

Moreover, in order to propose feasible LMI-based stability condition, we must rely on assumption (H2) about neural activation functions. Based on (H2), the following matrix inequalities hold for any positive diagonal matrices $L_{m}, T_{m}$

$$
\begin{aligned}
0 \leq & -y(t)^{T} L_{m} \Lambda_{1} y(t)+2 y(t)^{T} L_{m} \Lambda_{2} g(y(t))-g(y(t))^{T} L_{m} g(y(t)), \\
0 \leq & -y\left(t-\tau_{m}(t)\right)^{T} T_{m} \Lambda_{1} y\left(t-\tau_{m}(t)\right) \\
& +2 y\left(t-\tau_{m}(t)\right)^{T} T_{m} \Lambda_{2} g\left(y\left(t-\tau_{m}(t)\right)\right)-g\left(y\left(t-\tau_{m}(t)\right)\right)^{T} T_{m} g\left(y\left(t-\tau_{m}(t)\right)\right) .
\end{aligned}
$$

Furthermore, the zero equality (3) also plays an important role in derive feasible stability criterion expressed in terms of linear matrix inequalities. From Eq. (3), the following equality is true for any positive diagonal matrix $Z_{m}=\operatorname{diag}\left\{z_{1 m}\right.$, $\left.z_{2 m}, \cdots, z_{n m}\right\}$ 


$$
\begin{aligned}
0= & 2 \sum_{i=1}^{n} \dot{y}_{i}(t) z_{i m}\left[-\dot{y}_{i}(t)-d_{i m} y_{i}(t)+\sum_{j=1}^{n} a_{i j m} g_{j}\left(y_{j}(t)\right)+\sum_{j=1}^{n} b_{i j m} g_{j}\left(y_{j}\left(t-\tau_{m}(t)\right)\right)\right. \\
& +\bigwedge_{j=1}^{n} \alpha_{i j} \int_{-\infty}^{t} k_{j}(t-s) f_{j}\left(y_{j}(s)+x_{j}^{*}\right) \mathrm{d} s-\bigwedge_{j=1}^{n} \alpha_{i j} \int_{-\infty}^{t} k_{j}(t-s) f_{j}\left(x_{j}^{*}\right) \mathrm{d} s \\
& \left.+\bigvee_{j=1}^{n} \beta_{i j} \int_{-\infty}^{t} k_{j}(t-s) f_{j}\left(y_{j}(s)+x_{j}^{*}\right) \mathrm{d} s-\bigvee_{j=1}^{n} \beta_{i j} \int_{-\infty}^{t} k_{j}(t-s) f_{j}\left(x_{j}^{*}\right) \mathrm{d} s\right] \\
= & 2 \dot{y}(t)^{T} Z_{m}\left\{-\dot{y}(t)-D_{m} y(t)+A_{m} g(y(t))+B_{m} g\left(y\left(t-\tau_{m}(t)\right)\right)\right\} \\
& +2 \sum_{i=1}^{n} z_{i m} \dot{y}_{i}(t)\left(\bigwedge_{j=1}^{n} \alpha_{i j} \int_{-\infty}^{t} k_{j}(t-s) f_{j}\left(y_{j}(s)+x_{j}^{*}\right) \mathrm{d} s\right. \\
& \left.-\bigwedge_{j=1}^{n} \alpha_{i j} f_{j}\left(x_{j}^{*}\right)+\bigvee_{j=1}^{n} \beta_{i j} \int_{-\infty}^{t} k_{j}(t-s) f_{j}\left(y_{j}(s)+x_{j}^{*}\right) \mathrm{d} s-\bigvee_{j=1}^{n} \beta_{i j} f_{j}\left(x_{j}^{*}\right)\right) .
\end{aligned}
$$

Similar to (20), we obtain the following inequalities for any positive diagonal matrix $G$

$$
\begin{aligned}
& 2 \sum_{i=1}^{n} z_{i m} \dot{y}_{i}(t)\left(\bigwedge_{j=1}^{n} \alpha_{i j} \int_{-\infty}^{t} k_{j}(t-s) f_{j}\left(y_{j}(s)+x_{j}^{*}\right) \mathrm{d} s-\bigwedge_{j=1}^{n} \alpha_{i j} \int_{-\infty}^{t} k_{j}(t-s) f_{j}\left(x_{j}^{*}\right) \mathrm{d} s\right. \\
& \quad+\bigvee_{j=1}^{n} \beta_{i j} \int_{-\infty}^{t} k_{j}(t-s) f_{j}\left(y_{j}(s)+x_{j}^{*}\right) \mathrm{d} s- \\
& \left.\quad-\bigvee_{j=1}^{n} \beta_{i j} \int_{-\infty}^{t} k_{j}(t-s) f_{j}\left(x_{j}^{*}\right) \mathrm{d} s\right) \\
& \leq n \dot{y}(t)^{T} Z_{m} \Upsilon G^{-1} \Upsilon Z_{m} \dot{y}(t)+\left(\int_{-\infty}^{t} k(t-s) g(y(s)) \mathrm{d} s\right)^{T} \\
& \quad G\left(\int_{-\infty}^{t} k(t-s) g(y(s)) \mathrm{d} s\right) .
\end{aligned}
$$

Based on $\mathfrak{L} V_{\text {im }}(t, y(t))(i=1, \ldots, 6)$ and aforementioned inequalities, we can derive a less conservative stability conditions expressed by means of matrix inequalities. Substituting (15)-(28) into (13) and taking mathematical expectation gives that

$$
\mathbb{E} \mathfrak{L} V_{m}\left(t, y_{t}\right)=\zeta(t)^{T} \bar{\Phi}_{m} \zeta(t), \quad t \in\left[t_{k-1}, t_{k}\right), k \in \mathbb{Z}_{+} .
$$

where

$$
\begin{aligned}
\bar{\Phi}_{m}= & \Phi_{m}+n \varpi_{1}^{T} P_{m} \Upsilon H^{-1} \Upsilon P_{m} \varpi_{1}+n \varpi_{7}^{T} Z_{m} \Upsilon G^{-1} \Upsilon Z_{m} \varpi_{7} \\
& +4 \max \left\{-\left(\varpi_{8}-\varpi_{2}\right)^{T} S_{m}\left(\varpi_{8}-\varpi_{2}\right),-\left(\varpi_{9}-\varpi_{3}\right)^{T} S_{m}\left(\varpi_{9}-\varpi_{3}\right)\right\} \\
& -\frac{\bar{\tau}_{m}-\tau_{m}(t)}{\bar{\tau}_{m}}\left(\varpi_{1}-\varpi_{2}\right)^{T} W_{4}\left(\varpi_{1}-\varpi_{2}\right)-\frac{\tau_{m}(t)}{\bar{\tau}_{m}}\left(\varpi_{2}-\varpi_{3}\right)^{T} W_{4}\left(\varpi_{2}-\varpi_{3}\right) .
\end{aligned}
$$




\section{Neural Network World 1/2016, 7-28}

As $\bar{\Phi}_{m}$ is a linear convex function of variable $\tau_{m}(t)$, inequality $\bar{\Phi}_{m}<0$ is difficult to be verified by Matlab LMI software. Therefore we must derive an inequality of constant matrix. Based on the linear convex combination technique [32], inequality $\bar{\Phi}_{m}<0$ is true if and only if the following two inequalities hold simultaneously

$$
\begin{aligned}
& \Phi_{m}+n \varpi_{1}^{T} P_{m} \Upsilon H^{-1} \Upsilon P_{m} \varpi_{1}+n \varpi_{7}^{T} Z_{m} \Upsilon G^{-1} \Upsilon Z_{m} \varpi_{7}-\left(\varpi_{1}-\varpi_{2}\right)^{T} W_{4}\left(\varpi_{1}-\varpi_{2}\right) \\
& \quad+4 \max \left\{-\left(\varpi_{8}-\varpi_{2}\right)^{T} S_{m}\left(\varpi_{8}-\varpi_{2}\right),-\left(\varpi_{9}-\varpi_{3}\right)^{T} S_{m}\left(\varpi_{9}-\varpi_{3}\right)\right\}<0, \\
& \Phi_{m}+n \varpi_{1}^{T} P_{m} \Upsilon H^{-1} \Upsilon P_{m} \varpi_{1}+n \varpi_{7}^{T} Z_{m} \Upsilon G^{-1} \Upsilon Z_{m} \varpi_{7}-\left(\varpi_{2}-\varpi_{3}\right)^{T} W_{4}\left(\varpi_{2}-\varpi_{3}\right) \\
& \quad+4 \max \left\{-\left(\varpi_{8}-\varpi_{2}\right)^{T} S_{m}\left(\varpi_{8}-\varpi_{2}\right),-\left(\varpi_{9}-\varpi_{3}\right)^{T} S_{m}\left(\varpi_{9}-\varpi_{3}\right)\right\}<0 .
\end{aligned}
$$

As inequalities (30) and (31) are forms with the inverses of matrices $G, H$, they are infeasible with Matlab LMI software. The Schur complement plays an important role in changing inequality with the inverses of matrices into linear matrix inequalities. From the well known Schur complement, we deduce that inequality $\bar{\Phi}_{m}<0$ is equivalent to inequalities (9) with $j, \nu=1,2$. Therefore, if inequalities $(9)(j, \nu=1,2)$ hold, then from (29) we derive that

$$
\mathbb{E} \mathfrak{L} V_{m}\left(t, y_{t}\right)<0, \quad \forall t \in\left[t_{k-1}, t_{k}\right), k \in \mathbb{Z}_{+}
$$

Inequality (32) implies that the mathematical expectation of the LyapunovKrasovskii functional $V_{m}\left(t, y_{t}\right)$ decreases at any continuous time $t \in\left[t_{k-1}, t_{k}\right), k \in$ $\mathbb{Z}_{+}$. Next, we will prove that $V_{m}\left(t, y_{t}\right)$ decreases at any discontinuous time $t=$ $t_{k}, k \in \mathbb{Z}_{+}$, that is $V_{m}\left(t_{k}, y\left(t_{k}\right)\right)<V_{m}\left(t_{k}^{-}, y\left(t_{k}^{-}\right)\right)$. When $t=t_{k}$, from the condition (H5), we have

$$
V_{m}\left(t_{k}, y\left(t_{k}\right)\right)=V_{m}\left(t_{k}^{-}, y\left(t_{k}^{-}\right)\right)+y\left(t_{k}^{-}\right)^{T}\left[\left(I-\Gamma_{k}\right)^{T} P_{m}\left(I-\Gamma_{k}\right)-P_{m}\right] y\left(t_{k}^{-}\right)
$$

From Eq. (33), in order to derive that $V_{m}\left(t, y_{t}\right)$ decreases at $t=t_{k}$, it is sufficient to prove $\left(I-\Gamma_{k}\right)^{T} P_{m}\left(I-\Gamma_{k}\right)-P_{m}>0$. Considering inequality (7), it follows that

$$
\left(\begin{array}{cc}
I & 0 \\
0 & P_{m}^{-1}
\end{array}\right)\left(\begin{array}{cc}
P_{m} & \left(I-\Gamma_{k}\right) P_{m} \\
* & P_{m}
\end{array}\right)\left(\begin{array}{cc}
I & 0 \\
0 & P_{m}^{-1}
\end{array}\right) \geq 0
$$

that is

$$
\left(\begin{array}{cc}
P_{m} & I-\Gamma_{k} \\
* & P_{m}^{-1}
\end{array}\right) \geq 0
$$

From the Schur complement, we have

$$
P_{m}-\left(I-\Gamma_{k}\right)^{T} P_{m}\left(I-\Gamma_{k}\right) \geq 0 .
$$

Combining (33) with (34), we can deduce that

$$
V_{m}\left(t_{k}, y\left(t_{k}\right)\right) \leq V_{m}\left(t_{k}^{-}, y\left(t_{k}^{-}\right)\right), \quad k \in \mathbb{Z}_{+} .
$$


In order to prove that the Lyapunov-Krasovskii functional $V_{m}\left(t, y_{t}\right)$ decreases at any discontinuous time $t=t_{k}$ for different modes, we must depend on inequality (8). By simple calculation, it can be verified from (8) that

$$
V_{m}\left(t_{k}, y\left(t_{k}\right)\right) \leq V_{l}\left(t_{k}^{-}, y\left(t_{k}^{-}\right)\right) .
$$

For $t \in\left[t_{k-1}, t_{k}\right], k \in \mathbb{Z}_{+}$, in view of (32) and (35), we have

$$
V_{m}\left(t_{k}, y\left(t_{k}\right)\right) \leq V_{l}\left(t_{k}^{-}, y\left(t_{k}^{-}\right)\right) \leq V_{l}\left(t_{k-1}, y\left(t_{k-1}\right)\right) .
$$

By the similar proof and Mathematical induction, we can derive that (36) is true for any $m, l, \eta(0)=\eta_{0} \in \mathcal{N}, k \in \mathbb{Z}_{+}$

$$
V_{m}\left(t_{k}, y\left(t_{k}\right)\right) \leq V_{l}\left(t_{k}^{-}, y\left(t_{k}^{-}\right)\right) \leq V_{l}\left(t_{k-1}, y\left(t_{k-1}\right)\right) \leq \cdots \leq V_{\eta_{0}}\left(t_{0}, y\left(t_{0}\right)\right) .
$$

Inequality (37) implies that the Lyapunov-Krasovskii functional decreases at any continuous or discontinuous time for the same or different modes. Therefore, the system (3) is asymptotically stable in mean square. This completes the proof of Theorem 1.

\section{Appendix II}

\section{Proof of Theorem 2}

Inspired by $[4,36]$, we construct a Lyapunov-Krasovskii functional in the following form

$$
V\left(t, y_{t}\right)=y(t)^{T} P y(t)+\sum_{i=1}^{4} V_{i}\left(t, y_{t}\right)
$$

where

$$
\begin{aligned}
& V_{1}\left(t, y_{t}\right)=\int_{t-\tau(t)}^{t}\left[y(s)^{T} \Lambda_{4} E \Lambda_{4} y(s)-g(y(s))^{T} E g(y(s))\right] \mathrm{d} s, \\
& V_{2}\left(t, y_{t}\right)=\int_{t-\tau(t)}^{t}\left[\begin{array}{c}
y(s) \\
g(y(s))-\Lambda_{3} y(s)
\end{array}\right]^{T}\left[\begin{array}{cc}
Q & X \\
* & R
\end{array}\right]\left[\begin{array}{c}
y(s) \\
g(y(s))-\Lambda_{3} y(s)
\end{array}\right] \mathrm{d} s, \\
& V_{3}\left(t, y_{t}\right)=\bar{\tau} \int_{t-\bar{\tau}}^{t} \int_{\theta}^{t} \dot{y}(s)^{T}\left(S+W_{4}\right) \dot{y}(s) \mathrm{d} s \mathrm{~d} \theta+\int_{t-\bar{\tau}}^{t} y(s)^{T} W_{5} y(s) \mathrm{d} s, \\
& V_{4}\left(t, y_{t}\right)=\sum_{j=1}^{n} u_{j} \int_{0}^{\infty} k_{j}(\theta) \int_{t-\theta}^{t} g_{j}^{2}\left(y_{j}(s)\right) \mathrm{d} s \mathrm{~d} \theta .
\end{aligned}
$$

Note that

$$
\begin{aligned}
\dot{V}_{1}\left(t, y_{t}\right)= & y(t)^{T} \Lambda_{4} E \Lambda_{4} y(t)-g(y(t))^{T} E g(y(t)) \\
& -[1-\dot{\tau}(t)]\left[y(t-\tau(t))^{T} \Lambda_{4} E \Lambda_{4} y(t-\tau(t))-g(y(t-\tau(t)))^{T}\right. \\
& E g(y(t-\tau(t)))],
\end{aligned}
$$




$$
\begin{aligned}
\dot{V}_{2}\left(t, y_{t}\right)= & {\left[\begin{array}{c}
y(t) \\
g(y(t))-\Lambda_{3} y(t)
\end{array}\right]^{T}\left[\begin{array}{cc}
Q & X \\
* & R
\end{array}\right]\left[\begin{array}{c}
y(t) \\
g(y(t))-\Lambda_{3} y(t)
\end{array}\right]-[1-\dot{\tau}(t)] } \\
\times & {\left[\begin{array}{c}
y(t-\tau(t)) \\
g(y(t-\tau(t)))-\Lambda_{3} y(t-\tau(t))
\end{array}\right]^{T}\left[\begin{array}{cc}
Q & X \\
* & R
\end{array}\right] } \\
& {\left[\begin{array}{c}
y(t-\tau(t)) \\
g(y(t-\tau(t)))-\Lambda_{3} y(t-\tau(t))
\end{array}\right], }
\end{aligned}
$$

following the same line as in Theorem 1, we can complete the proof of Theorem 2.

\section{Acknowledgement}

This work was supported by the National Natural Science Foundation of China No. 61273022.

\section{References}

[1] BALASUBRAMANIAM P., VEMBARASAN V. Robust stability of uncertain fuzzy BAM neural networks of neutral-type with Markovian jumping parameters and impulses. Comput. Math. Applic. 2011, 62(4), pp. 1838-1861, doi: 10.1016/j.camwa.2011.06.027.

[2] CHEN Y., ZHENG W.X. Stability analysis of time-delay neural networks subject to stochastic perturbations. IEEE Trans. Systems Man Cyber., B, Cyber. 2013, 43(6), pp. 2122-2134, doi: 10.1109/TCYB. 2013.2240451.

[3] DONG M., ZHANG H., WANG Y. Dynamics analysis of impulsive stochastic CohenGrossberg neural networks with Markovian jumping and mixed time delays. Neurocomputing. 2009, 72(7-9), pp. 1999-2004, doi: 10.1016/j.neucom.2008.12.007.

[4] GE C., HUA C., GUAN X. New delay-dependent stability criteria for neural networks with time-varying delay using delay-decomposition approach. IEEE Trans. Neural Netw. Learn. Syst. 2014, 25(7), pp. 1378-1383, doi: 10.1109/TNNLS. 2013.2285564.

[5] HAN W., LIU Y., WANG L. Global exponential stability of delayed fuzzy cellular neural networks with Markovian jumping parameters. Neural Comput. \& Applic. 2012, 21(1), pp. 67-72, doi: $10.1007 / \mathrm{s} 00521-011-0685-4$.

[6] HUANG T., HUANG Y., LI C. Stability of periodic solution in fuzzy BAM neural networks with finite distributed delays. Neurocomputing. 2008, 71(16-18), pp. 3064-3069, doi: 10 . 1016/j. neucom. 2008.04.021.

[7] KWON O.M., PARK J.H., LEE S.M., CHA E.J. Analysis on delay-dependent stability for neural networks with time-varying delays. Neurocomputing. 2013, 103, pp. 114-120, doi: 10 . 1016/j.neucom. 2012.09.012.

[8] LIU Z., YU J., XU D. Vector Wirtinger-type inequality and the stability analysis of delayed neural network. Commun. Nonlinear Sci. Numer. Simulat. 2013, 18(5), pp. 1246-1257, doi: 10.1016/j.cnsns. 2012.09.027.

[9] LIU Z., YU J., XU D., PENG D. Triple-integral method for the stability analysis of delayed neural networks. Neurocomputing. 2013, 99, pp. 283-289, doi: 10.1016/j.neucom.2012.07. 005.

[10] LIU Z., ZHANG H., ZHANG Q. Novel stability analysis for recurrent neural networks with multiple delays via line integral-type L-K functional. IEEE Trans. Neural Netw. 2010, 21(11), pp. 1710-1718, doi: 10.1109/TNN.2010.2054107.

[11] LIU Z., ZHANG H., WANG Z. Novel stability criterions of a new fuzzy cellular neural networks with time-varying delays. Neurocomputing. 2009, 72(4-6), pp. 1056-1064, doi: 10. $1016 / j$.neucom. 2008.04 .001 . 


\section{Zhang Y., Zheng C.-D.: Novel stochastic stability conditions of fuzzy...}

[12] LOU X., CUI B. Delay-dependent stochastic stability of delayed Hopfield neural networks with Markovian jump parameters. J. Math. Anal. Appl. 2007, 328(1), pp. 316-326, doi: 10. 1016/j.jmaa. 2006.05.041.

[13] LU R., LI H., ZHU Y. Quantized $\mathrm{H}_{\infty}$ filtering for singular time-varying delay systems with unreliable communication channel. Circuits Syst. Signal Process. 2012, 31(2), pp. 521-538, doi: $10.1007 / \mathrm{s} 00034-011-9333-6$.

[14] LU R., WU H., BAI J. New delay-dependent robust stability criteria for uncertain neutral systems with mixed delays. J. Franklin Inst. 2014, 351(3), pp. 1386-1399, doi: 10.1016/j. jfranklin.2013.11.001.

[15] LU R., XU Y., XUE A. $\mathrm{H}_{\infty}$ filtering for singular systems with communication delays. Signal Process. 2010, 90(4), pp. 1240-1248, doi: 10.1016/j.sigpro.2009.10.007.

[16] POZNYAK A.S., SANCHEZ E.N. Nonlinear systems approximation by neural networks: Error stability analysis. Intell. Automat. Soft Compt. Int. J. 1995, 1(1), pp. 247-258.

[17] SUN J., LIU G.P., CHEN J., REES D. Improved delay-range-dependent stability criteria for linear systems with time-varying delays. Automatica. 2010, 46(2), pp. 466-470, doi: 10 . 1016/j. automatica. 2009.11.002.

[18] WANG Z., ZHANG H., JIANG B. LMI-based approach for global asymptotic stability analysis of recurrent neural networks with various delays and structures. IEEE Trans. Neural Netw. 2011, 22(7), pp. 1032-1045, doi: 10.1109/TNN.2011.2131679.

[19] WANG Z., ZHANG H., LI P. An LMI approach to stability analysis of reaction-diffusion Cohen-Grossberg neural networks concerning Dirichlet boundary conditions and distributed delays. IEEE Trans. Systems Man Cyber., B, Cyber. 2010, 40(6), pp. 1596-1606, doi: 10. 1109/TSMCB. 2010.2043095.

[20] WANG Z., LIU Y., YU L., LIU X. Exponential stability of delayed recurrent neural networks with Markovian jumping parameters. Phys. Lett. A. 2006, 356(4-5), pp. 346-352, doi: 10. 1016/j.physleta.2006.03.078.

[21] XU D., YANG Z. Impulsive delay differential inequality and stability of neural networks. $J$. Math. Anal. Appl. 2005, 305(1), pp. 107-120, doi: 10.1016/j.jmaa.2004.10.040.

[22] YANG T., YANG L. The global stability of fuzzy cellular neural networks. IEEE Trans. Circuits Syst. I. 1996, 43(10), pp. 880-883, doi: 10.1109/81.538999.

[23] ZHANG H., LIU Z., HUANG G.B. Novel delay-dependent robust stability analysis for switched neutral-type neural networks with time-varying delays via SC technique. IEEE Trans. Systems Man Cyber., B, Cyber. 2010, 40(6), pp. 1480-1491, doi: 10.1109/TSMCB. 2010.2040274

[24] ZHANG H., LIU Z., HUANG G.B., WANG Z. Novel weighting-delay-based stability criteria for recurrent neural networks with time-varying delay. IEEE Trans. Neural Netw. 2010, 21(1), pp. 91-106, doi: 10.1109/TNN.2009.2034742.

[25] ZHANG H., LUN S., LIU D. Fuzzy $\mathcal{H}_{\infty}$ filter design for a class of nonlinear discrete-time systems with multiple time delay's. IEEE Trans. Fuzzy Syst. 2007, 15(3), pp. 453-469, doi: 10.1109/TFUZZ.2006.889841.

[26] ZHANG H., MA T., HUANG G. B., WANG Z. Robust global exponential synchronization of uncertain chaotic delayed neural networks via dual-stage impulsive control. IEEE Trans. Systems Man Cyber., B, Cyber. 2010, 40(3), pp. 831-844, doi: 10.1109/TSMCB. 2009.2030506.

[27] ZHANG H., CAI L., BIEN Z. A fuzzy basis function vector-based multivariable adaptive controller for nonlinear systems. IEEE Trans. Systems Man Cyber., B, Cyber. 2000, 30(1), pp. $210-217$, doi: $10.1109 / 3477.826963$.

[28] ZHANG H., YANG F., LIU X., ZHANG Q. Stability analysis for neural networks with timevarying delay based on quadratic convex combination. IEEE Trans. Neural Netw. Learn. Syst. 2013, 24(4), pp. 513-521, doi: 10.1109/TNNLS.2012.2236571.

[29] ZHANG H., WANG Y. Stability analysis of Markovian jumping stochastic Cohen-Grossberg neural networks with mixed time delays. IEEE Trans. Neural Netw. 2008, 19(2), pp. 366-370, doi: 10.1109/TNN.2007.910738. 
[30] ZHANG H., WANG Z., LIU D. Global asymptotic stability of recurrent neural networks with multiple time-varying delays. IEEE Trans. Neural Netw. 2008, 19(5), pp. 855-873, doi: 10.1109/TNN. 2007.912319.

[31] ZHANG Y., SUN J. Stability of impulsive neural networks with time delays. Phys. Lett. A. 2005, 348(1), pp. 44-50, doi: 10.1016/j.physleta.2005.08.030.

[32] ZHANG Y., YUE D., TIAN E. New stability criteria of neural networks with interval timevarying delay: A piecewise delay method. Appl. Math. Comput. 2009, 208(1), pp. 249-259, doi: $10.1016 / \mathrm{j}$. amc. 2008.11.046.

[33] ZHENG C.-D., SHAN Q.-H., WANG Z. Improved stability results for stochastic CohenGrossberg neural networks with discrete and distributed delays. Neural Process. Lett. 2012, 35(2), pp. 103-129, doi: 10.1007/s11063-011-9206-9.

[34] ZHENG C.-D., SHAN Q.-H., ZHANG H., WANG Z. On stabilization of stochastic CohenGrossberg neural networks with mode-dependent mixed time-delays and markovian switching. IEEE Trans. Neural Netw. Learn. Syst. 2013, 24(5), pp. 800-811, doi: 10.1109/TnNLS. 2013.2244613.

[35] ZHENG C.-D., ZHANG Y., WANG Z. Novel stability condition of stochastic fuzzy neural networks with Markovian jumping under impulsive perturbations. Int. J. Mach. Learn. \& Cyber. 2014, pp. 1-9, doi: 10.1007/s13042-014-0298-1.

[36] ZHOU X., TIAN J., MA H., ZHONG S. Improved delay-dependent stability criteria for recurrent neural networks with time-varying delays. Neurocomputing. 2014, 129, pp. 401408, doi: $10.1016 / j$.neucom.2013.09.019. 